\title{
Optical periodicity analysis for radio selected BL Lacertae objects (RBLs) ${ }^{\star}$
}

\author{
J. H. Fan ${ }^{1,2,3}$, R. G. Lin $^{2}$, G. Z. Xie ${ }^{4}$, L. Zhang ${ }^{3}$, D. C. $\mathrm{Mei}^{3}$, C. Y. Su ${ }^{5}$, and Z. M. Peng ${ }^{3}$ \\ 1 National Astronomical Observatory, Chinese Academy of Sciences, Beijing, PR China \\ 2 Center for Astrophysics, Guangzhou University, Guangzhou 510400, PR China \\ 3 Department of Physics, Yunnan University, Kunming, PR China \\ 4 Yunnan Observatory, Chinese Academy of Science, Kunming 650011, PR China \\ 5 Department of Physics, Guangdong Industry University, Guangzhou, PR China \\ Received 15 December 1999 / Accepted 9 August 2001
}

\begin{abstract}
The recently compiled historical lightcurves of 10 RBLs are probed with the Jurkevich method in a search for periodic behaviour. Monte Carlo simulations of artificial lightcurves are used to detect spurious periods and evaluate sources of error in this kind of analysis. Additional analysis based on the discrete correlation function $(\mathrm{DCF})$ provides confirmation for those periods that could have physical significance. We find that periods in the range 1.4-17.85 yr are present in nine objects of the sample.
\end{abstract}

Key words. methods: data analysis - galaxies: BL Lacertae objects: general - galaxies: photometry

\section{Introduction}

The long-term optical variations of AGNs have been discussed in numerous papers (e.g. Webb et al. 1988; Pica et al. 1988; Bozyan et al. 1990; Stickel et al. 1993; Takalo 1994 and references therein). Some long-term optical variations have been claimed to be periodic (see Fan et al. 1997, 1998a, 1999, 2000; Lainela et al. 1999; and reference therein).

BL Lacertae objects are an extreme subclass of AGNs, which show rapid and high amplitude variability, high and variable polarization, weak emission line features $(E W<5 \AA)$ or none at all. There are two subclasses of BL Lacertae objects, namely, the radio-selected BL Lacertae objects (RBLs) and the X-ray selected BL Lacertae objects (XBLs). There are differences between these two subclasses from the observational point of view (see Padovani \& Urry 1995; Fan 1999a; Qin \& Xie 1998; Urry 1999). Based on the available data, some researchers have claimed periodicities in the light curves of some of these objects. The 11.65-year period (Sillanpää et al. 1988; Kidger et al. 1992) reported in the light curve of OJ287 was the primary motivation for the OJ-94 program and the prediction was confirmed (see Sillanpää et al. 1996a,b). The periodicity in the light curves can be used to predict new outbursts. This kind of periodicity may also shed some light on the mechanism at work in these objects.

Send offprint requests to: J. H. Fan,

e-mail: jhfan@guangztc.edu.cn

* Figures 2 to 10 are only available in electronic form at http://www.edpsciences.org
We have compiled an optical database for radioselected BL Lacertae objects (Fan \& Lin 2000a), which can be used to search for periodicity in the light curves. This paper is arranged as follows: in Sect. 2, we introduce Jurkevich method and present the results; in Sect. 3, we give some discussion, and finally in Sect. 4 , we provide a brief conclusion.

\section{Periodicity analysis}

\subsection{Jurkevich method}

We use the Jurkevich (1971) method to search for the periodicity in the light curve of several BL Lacertae objects. The Jurkevich method (Jurkevich 1971) is based on the expected mean square deviation (also see Fan et al. 1998a). It is less inclined to generate spurious periodicities than a Fourier analysis. It tests a run of trial periods around which the data are folded. All data are assigned to $m$ groups according to their phases around each trial period. The variance $V_{i}^{2}$ for each group and the sum $V_{\mathrm{m}}^{2}$ of all groups are computed. If a trial period equals the true one, then $V_{\mathrm{m}}^{2}$ reaches its minimum. So, a "good" period will give a much reduced variance relative to those given by other spurious trial periods and with almost constant values. Kidger et al. (1992) introduced a fraction of the variance $f=\frac{1-V_{\mathrm{m}}^{2}}{V_{\mathrm{m}}^{2}}$, where $V_{\mathrm{m}}^{2}$ is the normalized value. In the normalized plot, a value of $V_{\mathrm{m}}^{2}=1$ means $f=0$ and hence there is no periodicity at all. The best periods can be identified from the plot: a value of $f \geq 0.5$ suggests that there is a very strong periodicity and a value of $f<0.25$ 

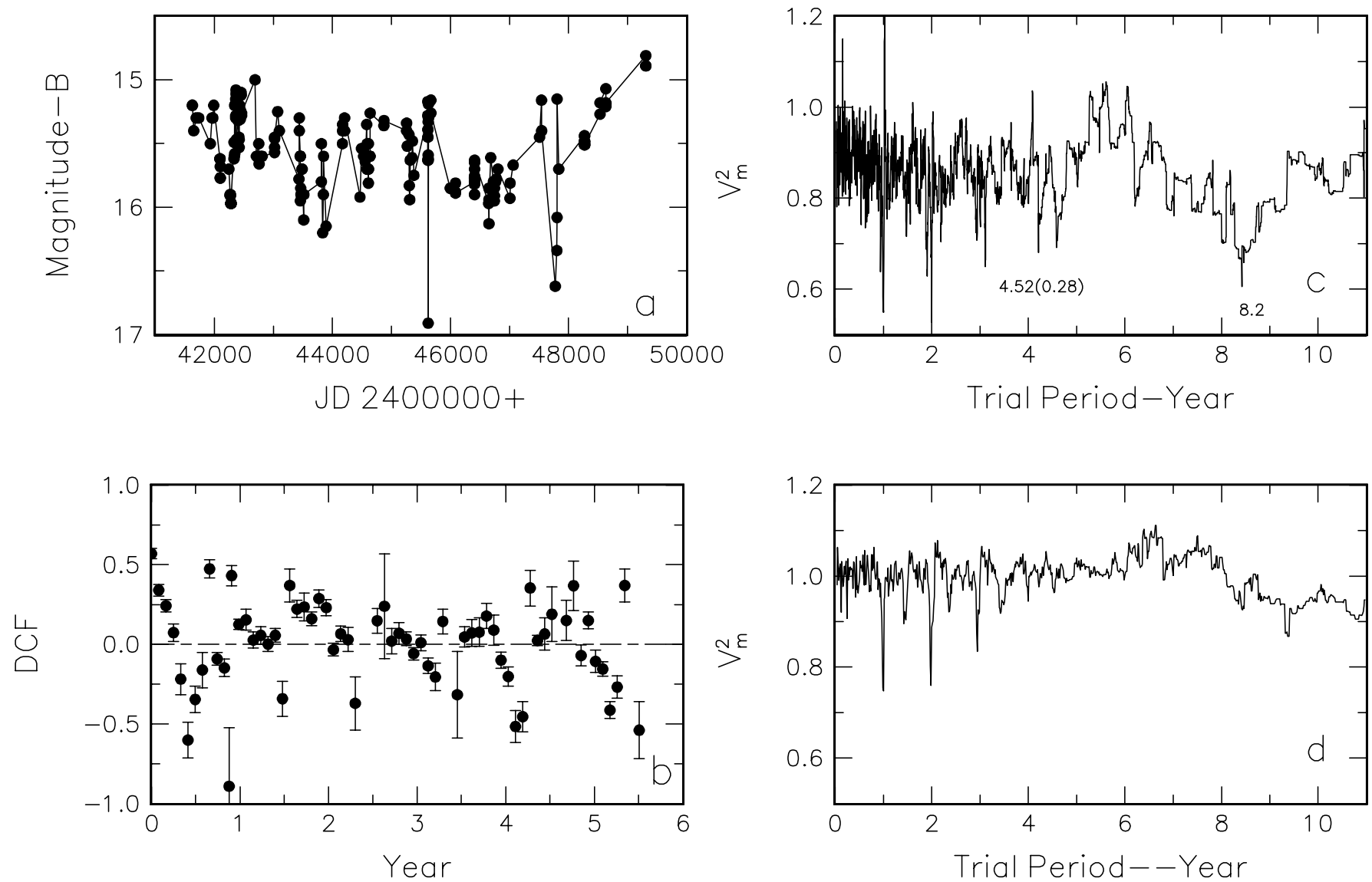

Fig. 1. a) Light curve, b) DCF result, c) plot of $V_{\mathrm{m}}^{2}$ vs. $P$ obtained from the measurements, d) plot of $V_{\mathrm{m}}^{2}$ vs. $P$ obtained from the Monte Carlo method for $0219+428$.

suggests that the periodicity, if genuine, is a weak one. A further test for the "spike"-shaped minimum is the relationship between the depth of the minimum and the noise in the "flat" section of the $V_{\mathrm{m}}^{2}$ curve close to the adopted period. If the absolute value of the relative change of the minimum to the "flat" section is large enough as compared with the standard error of this "flat" section, say ten times, the periodicity in the data can be considered as significant and the minimum as highly reliable (Kidger et al. 1992). Here we consider the half width at half minimum $(H W H M)$ as the "formal" error, as did Jurkevich (1971) and Fan et al. (1998a).

\subsection{Analysis}

Since there are more data in the $B$ band for most objects, we have used the $B$-band photometry, which is presented in Figs. 1a to 10a for each object, to investigate the longterm period of variation. In order to avoid the problem that sampling was more frequent during recent decades, we averaged the photometry into 5 day intervals. This is short enough compared to the long-term period (years), and thus unlikely to significantly distort the long-term variations. In our analysis, some early data are estimated from the relevant figures published in the literature (e.g. Miller 1978 for $1418+546$ ).
In the Jurkevich method, the calculation of the variance depends on the selection of the group number, $m$. This number $m$ has been chosen in this work in such a way that there are at least 20 pairs in each group. The linear trend is removed from the data set before analyzing the periodicities.

When the Jurkevich method is used to analyze the 5-day averaged data, the results are shown in Figs. 1c to $10 \mathrm{c}$. It is interesting to note that there is a common period of $\sim 1$-year for each object, which is probably due to annual Solar motion, seen also in the Monte Carlo analysis presented below.

\subsection{Monte Carlo result}

In the Jurkevich method analysis, it is possible that the distribution of the measurements, particularly for the data concentrating in groups, can give spurious periods, which are equal to the time interval between the data groups. So, it is necessary to rule out the spurious period. To do so, we use the Monte Carlo method to produce randomly "synthetic" measurements and then we apply the Jurkevich method to the "synthetic" measurements in order to identify spurious results. If the trial period obtained from the true measurements is also presented in the results obtained from the synthetic "measurements", then the period is likely caused by the distribution and should 
Table 1. Investigation results of periodicity of 10 RBLs

\begin{tabular}{cccc}
\hline $\begin{array}{c}\text { Name } \\
(1)\end{array}$ & $\begin{array}{c}P_{1} \\
(2)\end{array}$ & $\begin{array}{c}P_{2} \\
(3)\end{array}$ & $\begin{array}{c}P_{\text {Sillanpaa }} \\
(4)\end{array}$ \\
\hline $0219+428$ & $4.52 \pm 0.28$ & & \\
$0235+164$ & $2.95 \pm 0.15$ & & \\
$0754+100$ & $3.0 \pm 0.35$ & 17.85 & \\
$0851+202$ & $5.53 \pm 0.15$ & $11.75 \pm 0.5$ & 11.65 \\
$1215+303$ & $4.45 \pm 0.05$ & $6.89 \pm 1.0$ & \\
$1219+285$ & $9.2 \pm 0.1$ & $14.85 \pm 1.55$ & \\
$1308+326$ & $1.4 \pm 0.3$ & & \\
$1418+546$ & - & & \\
$1514-241$ & $2.0 \pm 0.2$ & & \\
$1807+698$ & $2.70 \pm 0.15$ & & \\
\hline \multicolumn{5}{c}{}
\end{tabular}

be discarded. By comparing the periods obtained from the real measurements and those from the "synthetic" measurements we can rule out the spurious periods caused by the data distributions.

The "synthetic" measurements are produced as follows: the times for the "synthetic" measurements for a given object are the real observations and the measurments are then randomly selected from the real data.

For each object, the periodicity analysis result obtained from the Monte Carlo method is presented in Figs. 1d to 10d. In the Jurkevich method, the spurious period likely shows up if the measurements are sampled group by group in the time axis. The intervals between the consecutive groups are the spurious period. It can be understood from the Jurkevich method since, in this case, there are no data in most bins out of the $m$ bins. Therefore, the calculation gives a reduced variance and the spurious period appears.

\subsection{DCF method}

The DCF (Discrete Correlation Function) method is intended for analyses of the correlation of two data set. It is described in detail by Edelson \& Krolik (1988) (also see Fan et al. 1998b). This method can indicate the correlation of two variable temporal series with a time lag, and can be applied to the periodicity analysis of a unique temporal data set. If there is a period, say $P$, in the lightcurve, then the DCF should show clearly that the data set is correlated with itself with time lags of $\tau=0$ and $\tau=P$ as presented in our previous paper (Fan \& Lin 2000b). We have implemented the method as follows.

Firstly, we have calculated the set of unbinned correlation $(U D C F)$ between data points in the two data streams $a$ and $b$, i.e.

$U D C F_{i j}=\frac{\left(a_{i}-\bar{a}\right) \times\left(b_{j}-\bar{b}\right)}{\sqrt{\sigma_{a}^{2} \times \sigma_{b}^{2}}}$,

where $a_{i}$ and $b_{j}$ are points in the data sets, $\bar{a}$ and $\bar{b}$ are the average values of the data sets, and $\sigma_{a}$ and $\sigma_{b}$ are the corresponding standard deviations. Secondly, we have averaged the points sharing the same time lag by binning the $U D C F_{i j}$ in suitably sized time-bins in order to get the $D C F$ for each time lag $\tau$ :

$D C F(\tau)=\frac{1}{M} \Sigma U D C F_{i j}(\tau)$,

where $M$ is the total number of pairs. The standard error for each bin is

$\sigma(\tau)=\frac{1}{M-1}\left\{\Sigma\left[U D C F_{i j}-D C F(\tau)\right]^{2}\right\}^{0.5}$.

The above process is performed for each object, the results are presented in Figs. 1b to 10b for each object.

We present below some remarks on the individual objects.

\subsection{Remark on the individual lightcurves}

From above analysis, we obtained the possibly physically significant periods for the objects and listed them in Table 1, in which Col. 1 gives the name of the source, Cols. 2 and 3 the periods found in the present paper, Col. 4 the period found by Sillanpää.

PKS $0219+428$ (3C 66A): There are optical B data available for more than 20 years for this object (Fig. 1a). A 65-day periodicity is claimed by Lainela et al. (1999). Our Jurkevich analysis gives periods of $0.99,2.0,3.1$, and $4.52 \pm 0.28$ years. The first three periods $0.99,2.0$ and 3.1 years, which are also present in the Monte Carlo result (see Fig. 1d), are spurious periods. The later two periods, 2.0 and 3.1 years should be the harmonics of the 1 -year period. The only possibly physically significant period is the $(4.52 \pm 0.28)$-year period with a $f=0.51$. When we employed the DCF method to study the same data, we found that there are correlation signatures with a time lag of 0.63 to $0.88,1.56$ to 1.94 , and 4.14 to 4.41 years (Fig. $1 \mathrm{~b}$ ). The (4.52 \pm 0.28$)$-year period does not conflict with the range of 4.14 to 4.41 by DCF analysis, and therefore can be taken as the physically significant period. There is also a broad minimum corresponding to a 8.2 -year period in Fig. 1c, which is as twice as the length of the period of 4.52-year and can be taken as the harmonics of the later.

AO 0235+164: It is violently variable in the entire electromagnetic spectrum (see Webb et al. 1988; Romero et al. 1997, 2000a; Kraus et al. 1999; Hartman et al. 1999). Our Jurkevich results based on the 16-year lightcurve indicate possible periods $(f<0.5)$ of 2.0 and $(2.95 \pm 0.15)$ years (Fig. 2c) and a strong period of 1.0 year. A possible $5.87 \pm 1.3$ year period can also be seen from the Jurkevich analysis, it is perhaps a harmonics of the $(2.95 \pm 0.15)$ year period. But from the results shown in Fig. 2d, the 1.0 and 2.0-year periods are possibly spurious ones. DCF analysis indicates that there is correlation with time lag of $\sim 3.2$ to 3.4 years (see Fig. $2 \mathrm{~b}$ ). Therefore, the $2.95 \pm 0.15$ year period does not conflict with the result by DCF method, and we can take the $(2.95 \pm 0.15)$-year period as the physically significant period in AO $0235+164$.

PKS 0754+100: There are about 82 years of available optical observations for this source (see Fig. 3a). 
The Jurkevich method shows a $17.85 \pm 1.3$ year period $(f=1.04)$. But if only the post-JD 2440000 data are taken into account, there are possible periods of 1.0, 2.0 and $3.0 \pm 0.35$ years (see Fig. 3c). Monte Carlo results indicate that the $3.0 \pm 0.35$ period is not caused by the data distribution (see Fig. 3d). There is also a broad minimum at $5.9 \pm 0.5$ years. These two periods $(3.0 \pm 0.35$ and $5.9 \pm 0.5)$ are likely the same with the later being the harmonic of the former. When DCF is adopted to the post-JD 2440000 data, there is a correlation signature with a time lag of 0.62 to 0.83 and 2.54 to 3.4 years (see Fig. 3b). The $(3.0 \pm 0.35)$-year period falls in the range of 2.54 to 3.4 years. The $3.0 \pm 0.35$ year period is consistent with the results by DCF method. Therefore there are possibly significant periods of $3.0 \pm 0.35$ and $(17.85 \pm 1.3)$ years in the light curve of PKS 0754+100.

PKS 0851+202 (OJ 287): Our results show periods of $5.53 \pm 0.15$ and $11.75 \pm 0.5$ years in the 104 year light curve. DCF method indicates that there is a correlation with time lags of 5.40 to 5.7 years and 10.11 to 11.48 years, which are consistent with the result claimed by Sillanpää et al. (1988). The results obtained by both the Jurkevich and DCF methods (see Figs. 4c and b) are consistent. Therefore, there are two periods, $5.53 \pm 0.15$ and $11.75 \pm$ 0.5 years in OJ 287.

PKS 1215+303 (ON 325): The Jurkevich method shows that there are possible periods of $0.99,2.0,(4.45 \pm$ $0.05)$ years, and a strong period of $(6.89 \pm 1.0)$ years in the 35 year light curve (see Figs. 5a,c). The $\sim 2.0$ year period is the harmonic of the 1.0-year period, and is spurious. The $(4.45 \pm 0.05)$ year period is not as obvious as the $(6.69 \pm 1.0)$ year period, but the Monte Carlo result shows that the $(4.45 \pm 0.05)$ and $(6.89 \pm 1.0)$ year periods are not caused by the data distribution, so that they should be physically significant. When DCF is applied to the optical data, correlation with time lags of 4.4 and 6.5 years is found (see Fig. 5b). So, the physically significant periods are the $4.45 \pm 0.05$ and $6.89 \pm 1.0$ year ones for PKS $1215+303$.

PKS 1219+285 (ON 231): There are periods of 1.0, $1.97,2.96,9.2 \pm 0.1,14.85 \pm 1.55,18.9 \pm 0.1$ years in the 105 year light curve (Fig. 6c). The periods of 1.97 and 2.96 years are likely the harmonics of 1 year, so the possibly significant periods are $9.2,14.85$ and 18.9 years. From the Monte Carlo result shown in Fig. 6d, we can see the 9.2-year period is not caused by the data distribution, but the 9.2 -year period is weak, i.e., $f<0.5$. The $14.85 \pm 1.55$ year period is consistent with the results obtained by the DCF method (see Fig. $6 \mathrm{~b}$ ), which shows that there is correlation with a time lag of 14.2 to 15.5 years. As for the 9.2 year period, there is a harmonic of the 18.9 year period, but the sign in Fig. $6 \mathrm{~b}$ is not clear. It should be discussed with new data. Therefore, there are possible $(9.2 \pm 0.1)$-year and $(14.85 \pm 1.55)$-year periods in the light curve of PKS $1219+285$.

PKS 1308+326: There are more than 15 years of observations in the $B$ band, which show possible periods of $1.0,1.4 \pm 0.3$, and $3.2 \pm 0.25$ years. As discussed for above objects, the 1.0 year period is a spurious one as shown in Figs. 7c and d. The DCF methods show correlation with time lag of 1.07 to 1.52 years (see Fig. 7b). The $1.4 \pm 0.3$ year period by Jurkevich method is in the range of 1.07 to 1.52 years by DCF method. As for the possible $(3.2 \pm 0.25)$-year period, it is likely the harmonic of the $(1.4 \pm 0.3)$-year period. So, there is a possible period of $1.4 \pm 0.3$ years in the light curve of PKS $1308+326$. But the period should be reviewed with more data since the data coverage in the present discussion is not long enough.

PKS 1418+546: The light curve covers a 88-year observation period, as shown in Fig. 8a, which concentrates in two groups. The latter group is fainter than the former one by 1.51 mag. To consider all the data, we added $1.51 \mathrm{mag}$ to the former group then adopted the Jurkevich method to the whole data, but there is no clear hint of periods (see Fig. 8). More data are needed to determine periods in the source.

PKS 1514-241: Our calculation is based on the light curve from 1972 to 1987 indicating strong periods of 1.0, $2.0 \pm 0.2$ years and a possible $3.9 \pm 0.25$ year period (Fig. 9c). There are two spurious periods: 1.0 and 2.0 years as shown in Fig. 9d. The broad minimum at 2 years includes some significant periods. A correlation with a time lag of 1.47 to 2.52 years is obtained by the DCF method (see Fig. 9b). Thus the $2.0 \pm 0.2$ year period is physically significant. The second minimum ( $3.9 \pm 0.25$-year) should be a harmonic of the $2.0 \pm 0.2$ year period.

PKS 1807+698 (3C 371): The light curve shown in Fig. 10a indicates that there is possibly a period of about 80 years in the light curve. Since there are only a few points before JD 2438000, we only performed the DCF and Jurkevich analysis on the post-JD 2438000 data and the Jurkevich result is shown in Fig. 10c. Jurkevich analysis gave an indication of a $2.7 \pm 0.15$-year period and the Monte Carlo results showed that the period is not caused by the data distribution as shown in Fig. 10d. When DCF was performed on the post-JD 2438000 data, there is correlation with a time lag of $0.42,1.95$ to 2.21 and 2.65 to 3.01 years (see Fig. 10b). This is consistent with the $(2.7 \pm 0.15)$-year period. But we should keep in mind that the data used for the period searching are too few for a definite argument of a period. New data are required.

\section{Discussion and conclusion}

Optical photometry has been obtained for about one hundred of years for some BL Lacertae objects. This long coverage makes it possible to search for periodicity in the light curves. Sillanpää et al. (1988) claimed that there is a 11.65-year period in OJ 287 and predicted that the next optical outburst would occur in the fall of 1994. Some other BL Lacertae objects are also claimed to show periodicity (see Fan et al. 1997, 1998a, 1999; Lainela et al. 1999).

To establish the reality of periods, we need to determine the duration of the data. The length of the data record required to demonstrate periodicity depends on 
the signal-to-noise ratio, systematic errors, regularity in sampling, nature of the measurements, and the nature of the underlying variation. Highly accurate data on a strictly periodic variable might require only 1.5 periods to yield an accurate measure of the period. But for some objects whose existing data are not sampled at regular intervals, a much longer time span of data must be used to demonstrate that a period is not simply a random event and probably has some physical significance (Kidger et al. 1992; Fan et al. 1997, 1998a). All the time coverages are longer than 1.5 times of the derived periods. Since we have discussed the periods for PKS 0735+178 (Fan et al. 1997) and BL Lacertae (Fan et al. 1998a) earlier, they are not included in the present paper.

Periodicity search is difficult because the astronomical observations are generally not sampled evenly. The distribution of measurements can cause spurious periods even using the Jurkevich method, which is less inclined to generate spurious ones than the Fourier analysis.

Some models have been proposed to explain longterm periodic variations in AGNs: the binary black hole model, the thermal instability model, and the perturbation model (e.g. Sillanpää et al. 1988; Meyer \& Meyer-Hofmeister 1984; Abraham \& Romero 1999; Fan et al. 2001). Although some of those models relate to the properties of the accretion disk, the thermal instability of the accretion disk is not good for the explanation of the period behaviour with timescales of a few years. The most promising models are the binary black hole model and the perturbation model. The helical jet related to the binary black holes have been used to explain the optical behaviour and objects (3C 345, OJ 287, BL Lacertae, and PKS $0735+178)$, thought to show periodicity in the historic light curves, also show helical trajectories of their VLBI radio components (Hardee 1987; Vicente et al. 1996; Tateyama et al. 1998; Gomez et al. 1999; Villata \& Raiteri 1999). In this sense, we would expect the similar VLBI observation results for other sources presented here.

In the present paper, the long-term optical $B$ light curves are used to both Jurkevich and DCF methods to search for periodicity for 10 RBLs (see Table 1). The Monte Carlo method is used to discard the spurious periods. For the possible significant periods, we folded the light curve. The derived possible periods are in the range of 1.4 to 17.85 years, as seen in Table 1. Villata \& Raiteri (1999) noticed that the radio components in the sources which display long-term periodicity in the optical light curves have helical trajectories, therefore, the frequent VLBI observations of the sources with long-term ( $\sim 10$ years) periods should be encouraged to detect the helical trajectories of the radio components in the sources. Besides, frequent VLBI observations of the sources with short periods should be encouraged in order to detect possible periodic ejection patterns, as was observed in 3C 273 by Abraham \& Romero (1999).

Acknowledgements. The authors are grateful to Dr. C. Bertout and the anonymous referee for the significant suggestions and comments and of Dr. G. E. Romero for his reading of the manuscript and suggestions. This work is supported by the National 973 Project of China (NKBRSF G19990754) and the National Natural Scientific Foundation of China (19973001, 19863001), Beijing Astrophysics Center, and the National Science Fund for Distinguished Young Scholars of China.

\section{References}

Abraham, Z., \& Romero, G. E. 1999, A\&A, 344, 61

Bozyan, E. P., Hemenway, P. D., \& Argue, A. N. 1990, AJ, 99, 1421

Fan, J. H., \& Lin, R. G. 2000a, ApJ, 537, 101

Fan, J. H., \& Lin, R. G. 2000b, A\&A, 355, 880

Fan, J. H., Romero, G., \& Lin, R. G. 2001, Acta Astron. Sin., 1,9

Fan, J. H., Xie, G. Z., Adam, G., et al. 1999, ASP Conf. Ser., 159,99

Fan, J. H. 1999a, ASP Conf. Ser., 159, 57

Fan, J. H. 1999b, MNRAS, 308, 1032

Fan, J. H., Xie, G. Z., Pecontal, E., et al. 1998a, ApJ, 507, 178

Fan, J. H., Adam, G. Z., Xie, G. Z., et al. 1998b, A\&AS, 133, 217

Fan, J. H., Xie, G. Z., Lin, R. G., et al. 1997, A\&AS, 125, 525

Gomez, J. L., Marascher, A. P., Alberdi, A., \& Gabuzda, D. C. 1999, ApJ, 519, 642

Hardee, P. E . 1987, ApJ, 318, 78

Hartman, R. C., Bertsch, D. L., Bloom, S. D., et al. 1999, ApJS, 123, 79

Jurkevich, I. 1971, Ap\&SS, 13, 154

Kidger, M. R., Takalo, L., \& Sillanpää, A. 1992, A\&A, 264, 32

Kraus, A., Quirrenbach, A., Lobanov, A. P., et al. 1999, A\&A, 344,807

Lainela, M., Takalo, L. O., Sillanpää, A., et al. 1999, ApJ, 521, 561

Meyer, F., \& Meyer-Hofmeister, E. 1984, A\&A, 132, 143

Miller, H. R. 1978, ApJ, 223, L67

Qin, Y. P., \& Xie, G. Z. 1998, ApJL, 508, 119

Romero, G. E., Combi, J. A., Benaglia, P., et al. 1997, A\&A, 326, 77

Romero, G. E., Cellone, S. A., \& Combi, J. A. 2000a, A\&A, 360, L47

Romero, G. E., Chajet, A., Abraham, Z., \& Fan, J. H. 2000b, A\&A, 360, 57

Sambruna, R., Maraschi, L., \& Urry, C. M. 1996, ApJ, 463, 444

Shen, B. S. P., \& Usher, P. D. 1970, Nature, 228, 1070

Sillanpää, A., Haarala, S., Valtonen, M. J., et al. 1988, ApJ, 325,628

Sillanpää, A., Takalo, L. O., Pursimo, T., et al. 1996a, A\&A, 305, L17

Sillanpää, A., Takalo, L. O., Pursimo, T., et al. 1996b, A\&A, 315, L13

Stickel, M., Fried, J. W., \& Kuhr, H. 1993, A\&AS, 98, 393

Takalo, L. O. 1994, Vistas Astron., 38, 77

Tateyama, C. E., Kingham, K. A., Kaufmann, P., et al. 1998, ApJ, 500, 810

Urry, C. M. 1999, ASP Conf. Ser., 159, 1

Urry, C. M., \& Padovani, P. 1995, PASP, 107, 803

Vicente, L., Charlot, P., \& Sol, H. 1996, A\&A, 312, 727

Villata, M., \& Raiteri, C. M. 1999, A\&A, 347, 30

Villata, M., Raiteri, C. M., Sillanpää, A., \& Takalo, L. O. 1998, MNRAS, 293, L13

Webb, J. R., Smith, A. G., Leacock, R. J., et al. 1988, AJ, 95, 374 